\title{
Interscalene Block Vs Suprascapular Block for Shoulder Arthroscopy - A Meta-Analysis
}

\author{
Andrew B. Maye, Eoghan T. Hurley, Cathal J. Moran, Gerard Curley
}

BACKGROUND: Shoulder arthroscopy is among the most commonly performed orthopaedic procedures. However, postoperative pain can result in increased morbidity, opioid consumption and decreased patient satisfaction. This study aims to meta-analyse the prospective cohort studies (PCS) in the literature to ascertain if interscalence block (ISB) or suprascapular block (SSB) for shoulder arthroscopy result in superior clinical outcomes. Our hypothesis was that ISB would result in decreased patient pain, and opioid consumption.

METHODS: A literature search was carried out according to the PRISMA guidelines. PCS comparing ISB and SSB were included. The outcomes measured were pain (VAS score), opioid consumption, complications and patient satisfaction. Statistical analysis was performed using Review Manager. A p-value of $<0.05$ was considered statistically significant.

RESULTS: Seven clinical trials were identified with 374 patients. In the immediate postoperative period in the PACU, there was a significantly lower pain score with ISB $(P=0.01)$. However, at 12 and 24 hours there was no significant difference between ISB and SSB ( $p$ $=0.08$, and $p=0.64$, respectively). There was significantly less opioid consumption in the first 24 hours with ISB ( $p=0.02$ ). There was no difference in the rate of complications or level of patient satisfaction between ISB and SSB ( $p>0.05$ for both).

DISCUSSION: The most important finding in this meta-analysis of 7 clinical trials was that there was a statistically significant result in favour of ISB with regards to pain in the immediate postoperative period combined with a statistically significant decrease in opioid consumption in the first 24 hours with ISB. Rising rates of opioid dependence are a significant issue in modern healthcare ${ }^{1}$. With mortality rates from opioid overdose a staggering 115 daily in the $U^{2}$, the potential benefit of ISB vs SSB in decreasing opioid usage in the first 24 hours cannot be ignored. The importance of developing strategies to limit opioid consumption in the postoperative period is undeniable ${ }^{3}$

\begin{abstract}
Even discounting the patient benefits from such strategies, the economic burden of opioid dependence alone justifies their use. The CDC estimates that the cost of prescription opioid addiction is $\$ 78.5$ billion annually in the US². Generally, decreased postoperative pain will increase patient satisfaction. In this case it is possible that the lack of improvement throughout the first 24 hours could account for similar patient satisfaction between both groups.
\end{abstract}

\begin{tabular}{|r|l|r|r|}
\hline OUTCOME & & FAVORS & P VALUE \\
\hline VAS in the PACU & MD ISB VS SSB (95\% CI) & ISB & 0.01 \\
\hline VAS at 12 hours & $\begin{array}{l}\text { MD ISB VS SSB (95\% } \\
\text { Cl) }\end{array}$ & Neither & 0.08 \\
\hline VAS at 24 hours & $\begin{array}{l}\text { MD ISB VS SSB (95\% } \\
\text { Cl) }\end{array}$ & Neither & 0.64 \\
\hline $\begin{array}{r}\text { Opioid usage in the first 24 } \\
\text { hours }\end{array}$ & $\begin{array}{l}\text { MD ISB VS SSB (95\% } \\
\text { Cl) }\end{array}$ & ISB & 0.02 \\
\hline Patient satisfaction & $\begin{array}{l}\text { MD ISB VS SSB (95\% } \\
\text { Cl) }\end{array}$ & Neither & 0.05 \\
\hline
\end{tabular}

CONCLUSION: ISB resulted in decreased pain levels in the immediate postoperative period and opioid consumption over the first 24 hours. There was no difference in the rate of complications or patient satisfaction between the two techniques.

1) Dart RC, Surratt HL, Cicero TJ, Parrino MW, Severtson SG, Bucher-Bartelson B, Green JL. Trends in Opioid Analgesic Abuse and Mortality in the United States. N Engl J Med 2015; 372:241-248.

2) CDC/NCHS, National Vital Statistics System, Mortality. CDC Wonder, Atlanta, GA: US Department of Health and Human Services, CDC; 2017. https://wonder.cdc.gov.

3) Halawi MJ, Lieberman JR. Opioids in Total Joint Arthroplasty: Moving Forward. J 